Check for updates

Cite this: RSC Adv., 2017, 7, 40834

\title{
Quantitative and multiplex dot-immunoassay using gap-enhanced Raman tags $\uparrow$
}

\author{
Boris Khlebtsov, (D) a Timophey Pylaev, (D) a Vitaly Khanadeev, (D) a Daniil Bratashov (D) b \\ and Nikolai Khlebtsov (D)*ab
}

Dot immunoassay has been proposed as a simple and fast method for detection of various analytes. However, this qualitative method has a narrow working range and is limited to multiplex detection. To overcome these drawbacks, we suggest a highly specific, quantitative, and multiplex dot immunoassay using plasmonic gap-enhanced Raman tags (GERTs) and a nitrocellulose membrane as a substrate. The assay principle is based on reading surface-enhanced Raman scattering (SERS) spectra from analyte drops on the membrane strip after incubation with GERTs conjugated to biospecific probes. Three types of GERTs were synthesised by embedding nitrobenzenethiol (NBT), naphthalenethiol (NT), and acetamidothiophenol (AcTP) Raman molecules inside Au core/shell nanoparticles. For proof-of-concept experiments, the NBT, NT, and AcTP GERTS were further functionalized with rabbit anti-rat, anti-human, and anti-chicken antibodies, respectively. For all three corresponding antigens, the detected Raman signals linearly depended on the analyte amount within the range from 10 to $300 \mathrm{ng}$. The multiplex capability of the assay is illustrated by simultaneous one-step determination of rat, human, and chicken IgGs with a mixture of functionalized GERTs by recording Raman maps for whole membrane to avoid the point-to-point repeatability problem. Thus, GERTs are promising SERS nanotags for advanced versions of immunoassays instead of common plasmonic SERS labels with Raman reporters excited by the external near field.

Received 23rd July 2017

Accepted 10th August 2017

DOI: 10.1039/c7ra08113h

rsc.li/rsc-advances
As an alternative to label-free approaches, SERS-tag sandwichbased immunoassay has been suggested ${ }^{6}$ as highly sensitive analytic tool. SERS tags are composed of a plasmonic nanoparticle modified with Raman active and targeting molecules ${ }^{7}$ (typically, monoclonal antibodies). The plasmonic core serves as the near field enhancer for Raman reporters, whereas the antibodies ensure selective binding to analyte molecules. Compared to most biopolymers, the reporter molecules have high Raman cross-section and fingerprint spectra with scattering lines located far from the lines of biomolecules to be detected.

A sandwich-based immunoassay include three basic steps: ${ }^{1}$ (1) functionalization of a substrate with capture antibodies to bind and concentrate analyte from a sample solution; (2) selective binding of captured analyte molecules to SERS tags; (3) readout and quantification of SERS signal from probe + target complex. Owing to high sensitivity and specificity, the sandwichbased SERS immunoassay has been widely used in clinical diagnostics, ${ }^{8}$ including detection of cancer biomarkers; ${ }^{9,10}$ influenza viruses; ${ }^{11}$ pathogenic bacteria; ${ }^{12}$ rheumatoid arthritis ${ }^{13}$ tuberculosis ${ }^{14}$ as well as drug,${ }^{15}$ food, ${ }^{16}$ and heavy metal ${ }^{17}$ control.

Along with the sandwich format, SERS immunoassay experiment may be performed in a simplest direct or dot immunoassay format, which is based on the specific staining of a sample drop adsorbed on a nitrocellulose membrane (NCM). ${ }^{18}$ At the visualization stage, the membrane is placed in a solution of
${ }^{a}$ Institute of Biochemistry and Physiology of Plants and Microorganisms, Russian Academy of Sciences, 13 Prospekt Entuziastov, Saratov 410049, Russia. E-mail: khlebtsov@ibppm.ru

${ }^{b}$ National Research Saratov State University, 83 Ulitsa Astrakhanskaya, Saratov 410012, Russia

$\dagger$ Electronic supplementary information (ESI) available. See DOI: 10.1039/c7ra08113h 
probes conjugated to labels, usually colloidal gold. ${ }^{19}$ The conjugates interact with targets and formed coloured spots on the membrane. The main drawback of dot immunoassay is low sensitivity as only small part of targets can interact with probes and contribute to the spot coloration. Nevertheless, due to rapidity and low cost, ${ }^{\mathbf{2 0 , 2 1}}$ the immunogold dot assay has been used for clinical diagnosis of various diseases (see, e.g., relevant references in review ${ }^{22}$ ).

Here, we are focused on fabrication and application of efficient SERS tags that can be used in both direct and sandwich immunoassay formats. We exploit the simplest direct immunoassay format known as dot immunoassay ${ }^{20}$ or "halfsandwich" immunoassay ${ }^{18}$ that employs NCM to capture and concentrate analyte from a one microliter solution.

The efficiency of SERS tags is determined by two factors: the enhancing properties of a plasmonic component and the intrinsic Raman cross-section of reporter molecules. Various types of $\mathrm{Au},{ }^{23} \mathrm{Au} @ \mathrm{Ag}^{24}$ nanoparticles and nanoparticle assemblies $^{25}$ have been developed and tested until now as SERS-active labels. Recently, new types of SERS tags were introduced, in which Raman reporters are embedded inside core/shell $\mathrm{Au}^{26,27}$ or $\mathrm{Au} @ \mathrm{Ag}^{28}$ nanostructures. Such SERS tags with embedded reporters were abbreviated as BRIGHTs $^{27}$ (bilayered Raman intense gold nanostructures with hidden tags), GERTs ${ }^{29}$ (gapenhanced Raman tags; GERT abbreviation will be used here), or Au $\mathrm{NMs}^{30}$ (Au nanomatryoshkas). Owing to high and uniform EM fields inside a nanometer or even subnanometer sized gap between the metallic core and shell, ${ }^{31,32}$ the overall enhancement of GERTs can be about $1.0 \times 10^{11}$, while the reporter molecules are protected from desorption and degradation by the outer metallic shell. GERTs have been successfully used for high-resolution and high-speed cell imaging, ${ }^{33}$ detection of biomolecules, ${ }^{34}$ and in vivo imaging. ${ }^{29}$ In addition to rational engineering of the plasmonic core, the overall performance of SERS tags can be greatly improved (up to two orders) through a properly selected molecules with high Raman cross sections. ${ }^{35}$

The use of efficient SERS tags instead of simple colloidal gold could improve the dot immunoassay performance by quantification of the results, decreasing the detection limit, and extension of concentration range. However, to the best of our knowledge, GERTs have not been applied to SERS immunoassay before, especially in a multiplex format. Besides, the optimal selection of Raman reporters for GERTs has also not been investigated previously.

In this work, we suggest a multiplex version of SERS dot immunoassay that utilizes three types of GERTs with nitrobenzenethiol, naphthalenethiol, and acetamidothiophenol reporter molecules embedded inside Au@Au core/shell nanoparticles. The fabricated GERTs were functionalized with antirat, anti-human and anti-chicken antibodies and used as labels in SERS dot immunoassay. Due to high and stable SERS signal from tags, we were able to record the reporter responses despite strong background Raman signals from NCM. As a result, the dot immunoassay was performed in a quantitative format with expanded range of linear detection. The multiplex capability of the assay is illustrated by a proof-of-concept experiment involving simultaneous one-step determination of target molecules (rat, human, and chicken IgGs) with a mixture of fabricated GERT conjugates. To fix the point-to-point repeatability problem, which is common for SERS detection on solid substrates, ${ }^{36}$ we recorded the whole Raman maps, thus measuring 2500 point spectra for each spot on NCM to obtain the representative average spectrum.

\section{Experimental section}

\section{Materials and reagents}

All chemicals were obtained commercially and used without additional purification. Cetyltrimethylammonium chloride (CTAC, 25\% water solution), L-ascorbic acid (AA, >99.9\%), benzenedithiol (BDT), 4-nitrobenzenethiol (NBT), 4-acetamidothiophenol (AcTP) and sodium borohydride $\left(\mathrm{NaBH}_{4}, 99 \%\right)$ were purchased from Sigma-Aldrich. The heterobifunctional cross-linker OPSS-PEG-NHS was purchased from Creative PEGWorks, USA. Hydrogen tetrachloroaurate trihydrate $\left(\mathrm{HAuCl}_{4}\right.$, 99.99\%) was purchased from Alfa Aesar. Ultrapure water obtained from a Milli-Q Integral 5 system was used in all experiments.

Lyophilized rat, human, and chicken IgG were purchased from Jackson ImmunoResearch, USA. Polyclonal rabbit antibodies to those IgG molecules (designated further anti-R, anti$\mathrm{H}$, and anti-Ch, respectively) were obtained from blood serum of immunized rabbits as described previously. ${ }^{37}$ The animals were housed under standard laboratory conditions, with access to food and water, ad libitum. Animal care and handling were in accordance with the Guide for the Care and Use of Laboratory Animals, the European Convention for the Protection of Vertebrate Animals Used for Experimental and Other Scientific Purposes, and the legislation of the Russian Federation. The experimental protocol was approved by the Committee for the Care and Use of Laboratory Animals at Saratov State University (Protocol H-147, 17.04.2001).

\section{GERT synthesis and conjugation with IgG}

At the first step, CTAC-capped $30 \mathrm{~nm}$ Au nanoparticles were synthesized by a seed-mediated protocol. ${ }^{30} \mathrm{~A}$ seed solution was prepared by mixing $10 \mathrm{~mL}$ of aqueous CTAC $(0.2 \mathrm{M}), 5 \mathrm{~mL} \mathrm{HAuCl}_{4}$ $(1 \mathrm{mM})$ with $800 \mu \mathrm{L}$ of a $\mathrm{NaBH}_{4}(0.01 \mathrm{M})$. Next, $30 \mathrm{~mL}$ of CTAC solution $(0.1 \mathrm{M})$ was mixed with $1.5 \mathrm{~mL}$ of $\mathrm{HAuCl}_{4}(10 \mathrm{mM})$, $300 \mu \mathrm{L}$ of ascorbic acid $(0.1 \mathrm{M})$, and $100 \mu \mathrm{L}$ of seeds. The obtained nanoparticles were functionalized with Raman reporters NBT, NT, and AcTP (designated further GERT1, GERT2, and GERT3, respectively). To this end, $10 \mathrm{~mL}$ of $\mathrm{Au}$ nanoparticles were mixed with $300 \mu \mathrm{L}$ of reporter solutions in ethanol $(2 \mathrm{mM})$ and kept undisturbed for 4 hours. The modified cores were then washed three times by centrifugation $(13000 \mathrm{~g}, 10 \mathrm{~min})$ to remove excess reagent, and finally dispersed in $5 \mathrm{~mL}$ of CTAC $(0.1 \mathrm{M})$.

At the next step, additional Au shell was grown on the surface of modified cores by adding $1.5 \mathrm{~mL}$ of Raman labelled nanoparticles into the growth solution consisting of $15 \mathrm{~mL}$ CTAC solution $(0.1 \mathrm{M}), 600 \mu \mathrm{L}$ ascorbic acid $(0.04 \mathrm{M})$, and $500 \mu \mathrm{L}$ of $\mathrm{HAuCl}_{4}(10 \mathrm{mM})$ under stirring. The GERTs were then washed two times by centrifugation at $5000 \mathrm{rpm}$ for 10 minutes and dispersed in $20 \mathrm{~mL}$ of water. 
GERT1, GERT2, and GERT3 particles were functionalized with anti-R, anti-H, and anti-Ch antibodies, respectively. First, OPSS-PEG-NHS cross-linker was reacted overnight with antibodies at a $10: 1$ molar ratio in $100 \mathrm{mM}$ sodium bicarbonate (pH 8.5). Modified antibodies were purified by gel filtration. Then, $200 \mu \mathrm{L}$ of OPSS-PEG-NHS-treated antibodies $\left(1 \mathrm{mg} \mathrm{mL}{ }^{-1}\right)$ was reacted with $20 \mathrm{~mL}$ of GERTs for $12 \mathrm{~h}$ to obtain functionalized conjugates. Finally, the bioconjugates were centrifuged at $4000 \mathrm{~g}$ for 10 minutes, and dispersed in $20 \mathrm{~mL}$ of $100 \mathrm{mM}$ PBS buffer ( $\mathrm{pH}=7.4)$.

Extinction spectra of as-prepared and functionalized $\mathrm{Au}$ nanoparticles were measured with a Specord BS-250 and Specord S-300 spectrophotometers (Analytik, Jena, Germany). Transmission electron microscopy (TEM) images were obtained using Libra-120 transmission electron microscope (Carl Zeiss, Jena, Germany) at the Simbioz Center for the Collective Use of Research Equipment in the Field of Physical-Chemical Biology and Nanobiotechnology at the IBPPM RAS.

\section{Dot immunoassay}

The dot assay was carried out on a $0.45 \mu \mathrm{m}$ NCM (Schleicher \& Schuell, Germany). $1 \mu \mathrm{L}$ of antigen (human, rat or chicken IgG) with concentrations ranging from 300 to $0.1 \mu \mathrm{g} \mathrm{mL}{ }^{-1}$ was spotted onto NCM strip. Scheme 1 shows three basic steps involved in a SERS dot immunoassay with GERT conjugates. During $60 \mathrm{~min}$ incubation period, the antigens were adsorbed onto membrane. To exclude nonspecific binding of GERTs, the membrane was further incubated in a blocking buffer containing $0.1 \%$ BSA, $150 \mathrm{mM} \mathrm{NaCl}$, and $20 \mathrm{mM}$ TrisHCl (pH 8.2) for $30 \mathrm{~min}$. On the visualization stage, the membrane was placed in a suspension of GERT-antibody conjugates (or in a mixture of GERT conjugates) for $1 \mathrm{~h}$.

The biospecific reaction could be observed visually as coloured spots. After incubation with conjugates, the membrane was rinsed with water and stored in water at $4{ }^{\circ} \mathrm{C}$ for further SERS experiments.

\section{SERS experiments}

Normal Raman spectra of reporter solutions (0.1 M in ethanol) and SERS spectra of GERT solutions were acquired with a Peak

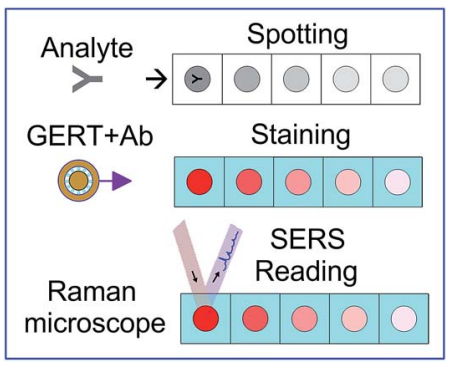

Scheme 1 Schematic illustration of three basic steps of a SERS dot immunoassay with GERT conjugates: (1) applying the target analyte drops; (2) staining the spots using GERT conjugates with probe molecules and blocking the unspotted membrane areas; (3) SERS mapping using specific reporter lines of GERTs.
Seeker Pro 785 Raman spectrometer (Ocean Optics) in $1 \mathrm{~cm}$ quartz cuvettes using $785 \mathrm{~nm}$ irradiation $(30 \mathrm{~mW})$. The acquisition interval was $30 \mathrm{~s}$ and all spectra were averaged over 10 independent runs.

SERS maps for NCM with biospecifically adsorbed GERT conjugates were obtained using Renishaw inVia Raman microscope $(785 \mathrm{~nm}$ ) equipped with $5 \times$ objective. Mapping area covered whole NCM with resolution of $100 \mu \mathrm{m}(150 \times$ 300 pixels). Integration time was $100 \mathrm{~ms}$ per each point. The principal component analysis (PCA) of spectra was done with default parameters in Chemometrics module of Renishaw WiRE4.2 software to separate specific peaks of GERTs from NCM background.

\section{Results and discussion}

\section{GERTs synthesis and characterization}

The fabrication of Au GERTs consists of three main steps schematically represented in Fig. 1: (1) synthesis of a gold core; (2) modification with reporter molecules; and (3) growth of an additional Au layer. Commonly, spherical Au nanoparticles with diameter of about $20 \mathrm{~nm}$ are used as a core for synthesis of GERTs. ${ }^{26-29}$ Recently, we demonstrated ${ }^{30}$ that the use of polygonal cores instead of spherical ones results in higher SERS response from gap-embedded reporters. A superior performance of such GERTs was explained ${ }^{30,31}$ by formation of bridged nanogaps and stronger internal EM fields as compared with EM fields inside hollow gaps formatted with smooth Au cores.

Further, the use of $35 \mathrm{~nm}$ Au cores also gives superior SERS enhancement compared to that for $15 \mathrm{~nm}$ cores. Based on these observations, we used here $30 \mathrm{~nm}$ polygonal $\mathrm{Au}$ cores for fabrication of GERTs.

Note that the difference between nanobridged and hollow gap structures was clearly revealed from HRTEM images only for small $15 \mathrm{~nm}$ cores and for BDT molecules with two thiolated groups. ${ }^{30}$ In this work, we did not consider this point because of poor resolution of subnanometer gaps in GERTs with $30 \mathrm{~nm}$ cores (similar observations have been reported previously ${ }^{\mathbf{3 0} 38}$ ). Besides, it is not clear at present whether the structural difference between GERTs with hollow and bridged nanogaps, as described in ref. 30, can be obtained for Raman reporters used in this work.

According to TEM analysis (Fig. 1A), the Au cores have polygonal shape with average size of $30 \pm 2 \mathrm{~nm}$. Their plasmonic extinction peak is located at $535 \mathrm{~nm}$ (curve 0, Fig. 1E) thus determining intense red colour of the suspension (left inset in Fig. 1E). On the second fabrication stage, Au cores were functionalized with thiolated aromatic molecules which serve both as Raman reporters and spacers. Note that in previous studies, BDT molecules were used for such purposes. ${ }^{27,28 d, 30,39,40}$ BDT has two thiol groups, so it was believed that BDT molecular structure promote formation of gap inside core/shell particles. Here, we demonstrate successful formation of gaps inside GERTs with the use of reporters possessing only one thiol group. Such an approach opens the way for advanced selection of Raman reporters with high cross sections and characteristic spectral lines. 


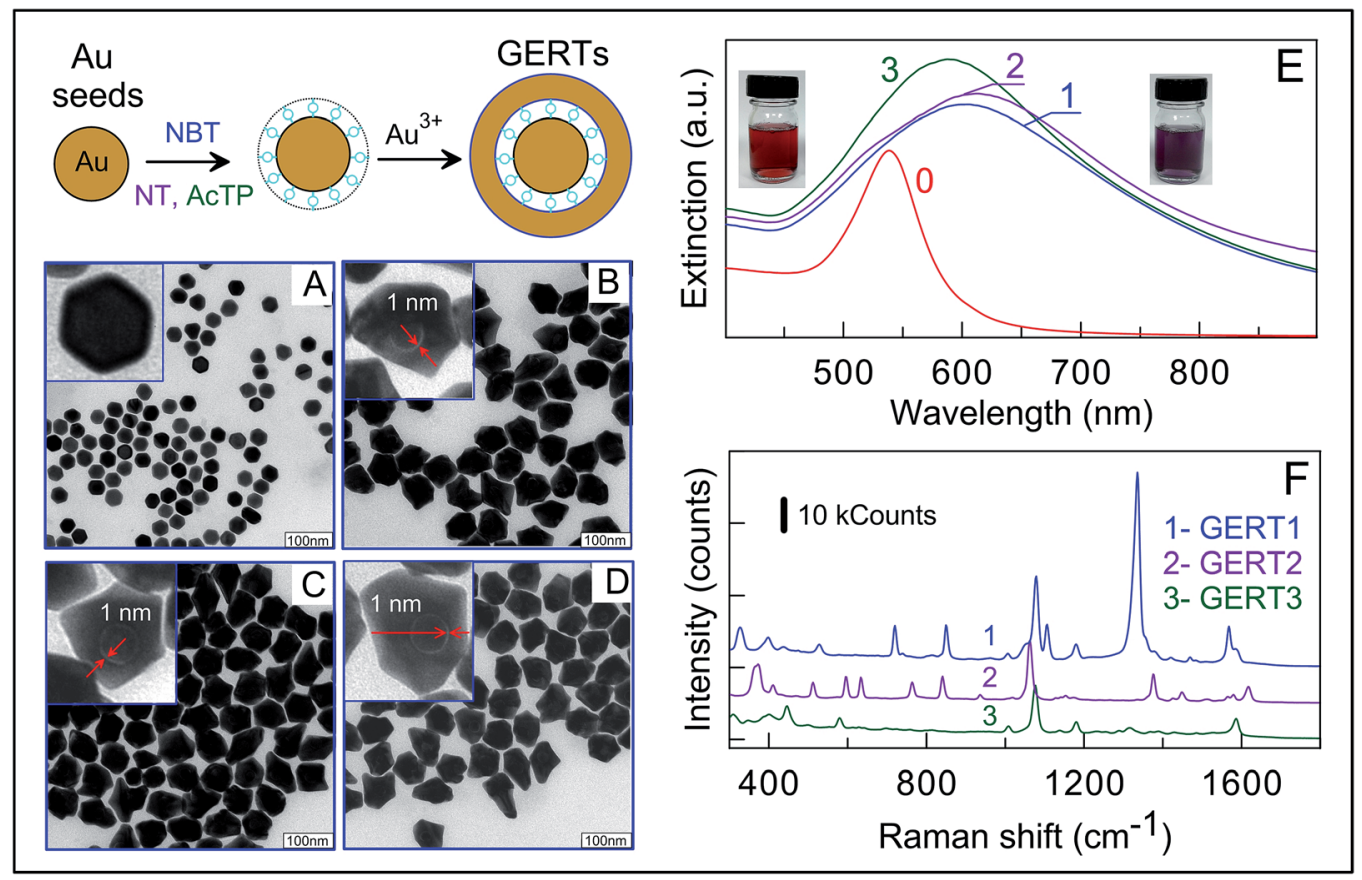

Fig. 1 Schematic representation of three-step synthesis of GERTs (left top picture). TEM image of Au polygonal cores (A) and GERT1, GERT2, GERT3 particles with embedded NBT (B), NT (C), and AcTP (D) molecules, respectively. All bars are 100 nm. The red arrows in enlarged insets (B-D) demonstrate $\sim 1 \mathrm{~nm}$ gaps inside GERTs. Panels (E and F) show extinction and SERS spectra of GERT1 (1), GERT2 (2), and GERT3 (3). The extinction spectrum ( 0 ) in the panel $(E)$ corresponds to Au polygonal cores. The left and right images in the panel $E$ display suspensions of polygonal Au cores (spectrum 0) and GERT1 tags (spectrum 1), respectively.

Fig. 1B-D represent typical overview and enlarged TEM images of GERTs fabricated with the use of AcTP, NT, and NBT molecules. For all three types of Raman reporters we observed the formation of nanogaps inside resulted nanoparticles. The size of this gap estimated by TEM imaging is about of $1 \mathrm{~nm}$, which is in line with previously reported data for dithiol aromatic molecules. ${ }^{30,31,38}$ The overgrowth of $\mathrm{Au}$ shell lead to increase in nanoparticle size up to $80 \pm 15 \mathrm{~nm}$, which corresponds to $25 \pm 5 \mathrm{~nm}$ shell thickness. Note the GERTs have similar morphology and size for all three types of reporters used. From optical point of view, the formation of GERTs results in shift of plasmon resonance from 535 to $580-600 \mathrm{~nm}$ and broadening the plasmonic peak (Fig. 1E). The colour of solutions changed from the initial red to purple. The red shift of the resonance peak is due to the increased particle size and the broadening the spectral peak can be most likely explained by the irregular particle shape and the size polydispersity.

To elucidate the dependence of SERS responses on the GERT reporter type, we measured SERS spectra of particles with different embedded molecules at a constant excitation wavelength of $785 \mathrm{~nm}$. Fig. 1F shows three experimental spectra acquired under the same experimental conditions and accumulation times. Characteristic Raman lines of reporter molecules are evidently seen in all spectra. For example, the SERS spectra of embedded NBT molecules (blue curve in Fig. 1F) are dominated $^{41}$ by the $\delta(\mathrm{CS}), \gamma(\mathrm{CCC}), \pi(\mathrm{CH})+\pi(\mathrm{CS})+\pi(\mathrm{CC}), \pi(\mathrm{CH})$, $\nu(\mathrm{CS}), \delta(\mathrm{CH}), \nu\left(\mathrm{NO}_{2}\right)$, and $\nu(\mathrm{CC})$ vibrations at $390,560,723,854$, 1081, 1110, and $1569 \mathrm{~cm}^{-1}$, respectively. It should be emphasized that the fingerprint SERS spectra of GERTs with different reporters allow performing the multiplex assay with a mixture of conjugates.

Direct comparison of peak amplitudes for three types of SERS tags gives strong evidence for advantaged SERS properties of NBT-based GERTs. In general, the intensity of SERS signal depends on the Raman cross-section of reporter molecules and the enhancing properties of plasmonic nanostructure. A typical figure of merit for the plasmonic enhancement of SERS platforms is the fundamental enhancement factor (EF), which is calculated as the ratio of the SERS intensity to the normal Raman intensity normalized to the number of excited molecules. $^{42}$ To calculate EF for our GERTs, the intensity of measured SERS spectra was compared with that for normal Raman spectra of reporters under identical experimental conditions. The details of calculations are given in the ESI. $\dagger$

The obtained EFs are $5.6 \times 10^{6}, 4.8 \times 10^{6}$, and $4.9 \times 10^{6}$ for NBT, NT, and AcTP molecules, respectively. These values are close to previously reported data for GERTs with embedded BDT molecules. ${ }^{30}$ Two important notes are in order here. First, the above EFs are higher than typical EFs for common SERS tags with Raman molecules attached to Au particle surface. Second, the obtained EFs were calculated assuming the monolayer coverage of reporters on the cores. This means that the above EF values are minimal estimations for the ensemble average. Taking into account similar structure of GERTs with different embedded reporters together with close EF values, on the one hand, and quite different normal Raman signals from reporter 
solutions, on the other, we assume the differences in SERS response to be totally caused by the difference in Raman crosssections of reporters. In particular, GERTs with NBT molecules have superior SERS performance.

For further use as labels for SERS dot assay, GERTs were conjugated to rabbit IgG (anti-chicken, anti-human, and antirat) by using the long-chain heterobifunctional cross-linker OPSS-PEG-NHS. This protocol has previously been used for successful conjugation of different $\mathrm{Au}^{\mathbf{4 3 , 4 4}}$ and $\mathrm{Au} @ \mathrm{Ag}$ nanoparticles. ${ }^{45}$

According to Scheme 1, the main difference between common and SERS immunoassays consists in SERS mapping of the membrane and quantification of the reaction outcome. In general, the assay can easily be multiplexed with GERT labels fabricated with different reporter molecules. For a proof-ofconcept illustration of SERS dot immunoassay, we chose three biospecific pairs (antigen-antibody): rat IgG (R-IgG)-anti-R, chicken IgG (Ch-IgG)-anti-Ch, and human IgG (H-IgG)-anti-H.

The panel (A) in Fig. 2 shows the assay results performed for sequential double dilutions of target antigens R-IgG, H-IgG, and Ch-IgG, which were examined with GERT1 + anti-R, GERT2 + anti-H, and GERT3 + anti-Ch conjugates. For all NMCs, the first spot corresponds to $300 \mu \mathrm{g} \mathrm{mL^{-1 }}$ antibody concentration; the further dots correspond to double dilutions of target molecules up to $0.6 \mu \mathrm{g} \mathrm{mL}{ }^{-1}$. The bottom rows of all the membranes show the application of diluted rabbit serum as a negative control; the concentration of rabbit IgG was $1 \mathrm{mg} \mathrm{mL}^{-1}$. For all negative control spots, not a single one was stained, thus indicating the absence of nonspecific binding. The naked eye examination results in the maximal detectable dilution about $1: 32$, which corresponds to app. $30 \mu \mathrm{g} \mathrm{mL} \mathrm{m}^{-1}$ minimal concentration. This limit of detection (LOD) agrees with the previously reported experimental $^{45}$ and theoretical ${ }^{46}$ estimations for the sensitivity of a silica/Au nanoshell and $\mathrm{Au} @ \mathrm{Ag}$ nanocages dot immunoassays.

The same dot spots were examined by the principal component (PCA) analysis, as shown in the panel (A). By contrast to identical pink-red colours of bright-field images, the PCA analysis reveals three different false-colour reaction outcomes for immunoassays with GERT1, GERT2, and GERT3 conjugates. In fact, the red, blue, and green spots on each NMC represent the first components in PCA decompositions of SERS spectra with $100 \mu \mathrm{m}$ step along each axes.

For illustrative purposes, panels (B, C, and D) in Fig. 2 provide characteristic SERS spectra measured for all three immunoassays. The light red, blue, and green bars select most intensive peaks at 1380,1067 , and $1081 \mathrm{~cm}^{-1}$. The numbers at spectra designate multiplier factors made for convenient comparison. The circles indicate spectra for maximal antigen dilutions $(1: 128,1: 64$, and $1: 32)$ for which the minimal SERS peak still can be resolved. In other words, these dilutions determine the LODs corresponding to $2.3,4.7$, and $9.4 \mu \mathrm{g} \mathrm{mL}^{-1}$ concentrations of anti-R, anti- $\mathrm{H}$, and anti-Ch antigens, respectively. In terms of analyte amount, the LODs are 2.3, 4.7, and 9.4 $\mathrm{ng}$, respectively. These values agree with reported data $^{23 b, 18}$ for SERS assay with NCM and gold/silica nanoshells as tags.

For quantitative examination of most intensive PCA peaks, one has to apply an appropriate averaging procedure. Indeed,

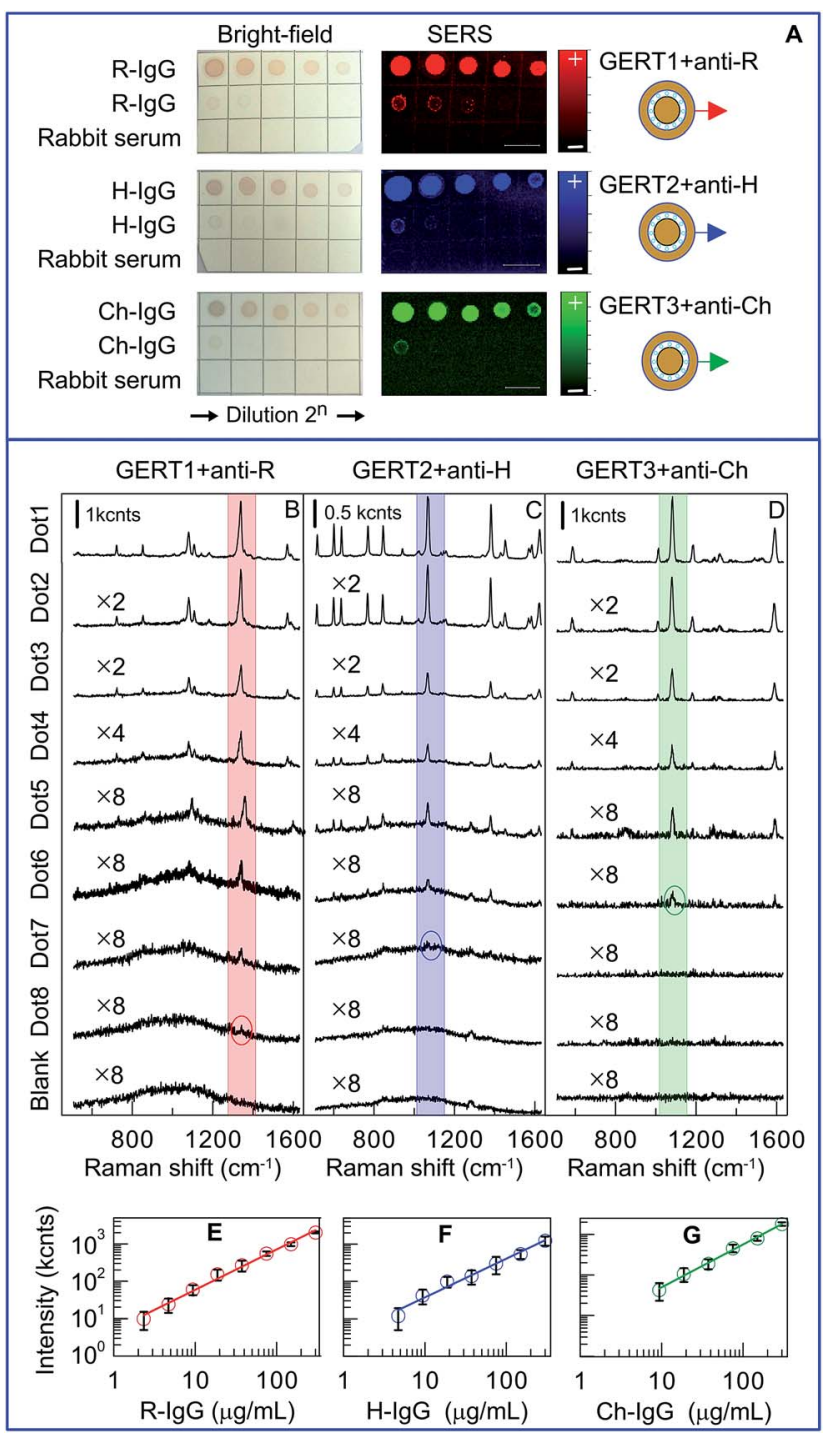

Fig. 2 SERS dot immunoassay with conjugates (A) GERT1 + anti-R, GERT2 + anti-H, and GERT3 + anti-Ch. The left column corresponds to the naked eye observation; the right column is the false-colour SERS maps of the same NCM (the first principal components PCA1 derived from Renishaw WiRE4.2 software). One microliter drops of analyte initial concentration, $300 \mu \mathrm{g} \mathrm{mL}^{-1}$; successive double dilutions, $1: 2^{n}$ were spotted onto a NCM in the center of drawn $5 \mathrm{~mm}$ squares. No staining and SERS signal are seen for rabbit serum used as a negative control. SERS spectra of dots onto membrane correspond to biospecific adsorption of GERT1 (B), GERT2 (C) and GERT3 (D). The numbers at spectra stand for multiplier factors introduced for convenient representation. Concentration-SERS intensity response curves for dot assays with GERT1 + anti-R (E), GERT2 + anti-H (F), and GERT3 + anti-Ch (G) conjugates.

the peak intensity distribution within each spots (about of $30 \times$ 36 pixel area) is strongly inhomogeneous because of inhomogeneous distribution of SERS tags. Similar situation has been reported for SERS mapping of lateral flow immunoassay (LFA) with hollow Au sphere SERS tags. ${ }^{47}$ There are several reasons for such inhomogeneous distribution. First, the hot spots in each pixel are not uniform because of different local concentration of SERS tags. Second, SERS response critically depends on the 
laser spot size. Crawford et al. ${ }^{\mathbf{4 8}}$ showed that for small laser spot sizes there are large probable deviation in the accuracy of SERS measurement. That is why we used here a $5 \times$ objective in contrast to $50 \times$ one used for LFA. $^{47}$ Finally, the structural properties of NTM or other substrate also can play important role.

In line with Hwang et al. approach, ${ }^{47}$ the point-to-point repeatability problem was resolved here by averaging the measured intensities for all 1080 pixel points. Such a procedure gives reproducible average intensities with much more statistical ensemble points as compared to previously reported data with 10 to 70 randomly selected points on SERS substrates. ${ }^{36,49} \mathrm{It}$ is this average intensity for each spot that was used for construction of calibration plots displayed in panels (E, F, and $\mathrm{G})$. The point-to-point repeatability as evaluated from Raman maps in terms of the normalized standard deviation (STD) for a particular SERS intensity is about \pm 0.3 for $10 \mathrm{ng}$ analyte and decreases for higher analyte amounts. According to our experimental observation (data not shown), the sample-to-sample reproducibility in terms of STD for major peak intensity is about $10 \%$ for GERT1 and increase to $15 \%$ for GERT3.

The calibration plots demonstrate exact linear correlations between the antigen amount and the SERS intensity; however the LODs and the linear calibration ranges differ notably for different GERTs. The panel (E) in Fig. 2 clearly demonstrates superior performance of GERTs with embedded NT Raman reporters in both the maximal linear response range (from 2 to $300 \mathrm{ng}$ ) and the LOD about $2 \mathrm{ng}$ of R-IgG. This observation points to the importance of appropriate choice of Raman reporters with maximal intrinsic cross section to ensure maximal overall performance of gap-enhanced SERS tags.

It should be noted here that in majority of published works $3,9 a, 9 e, 13 b, 14,23 d, 23 e, 24 b, 47,50$ the reported calibration curves (SERS response $v s$. analyte concentration) were referred to as linear dependences. However, a close inspection reveal the linear (or even saturated) correlation between SERS intensity and logarithm of concentration $I_{\text {SERS }}=a+b \times \log (c)$ (the work ${ }^{9 c}$ is a rare exception where the power $\log \left(I_{\text {SERS }}\right)=a+b \times \log (c)$ plots was demonstrated). In contrast to published reports, all plots in panels (E, F, and $\mathrm{G}$ ) do display linear fits.

The second important point to be discussed here is the low sensitivity of direct immunoassay format. Indeed, direct comparison the above nanogram LODs with reported concentration LODs (ranging from $1 \mu \mathrm{g} \mathrm{mL}^{-1}$ to fg $\mathrm{mL}^{-1}$ (ref. $23 d$ and $50 c$ )) definitely shows that the immunoassay format (direct $v s$. sandwich) is the crucial factor determining the overall assay sensitivity. The main drawback of the direct immunoassay is very small efficiency of biospecific binding of tags to targets as most of analyte molecules are hidden by porous structure of NTM. Therefore, one has to apply significant excess of analyte for each spot to ensure acceptable SERS readout. Quite similarly, even in most advanced sandwich immunoassay formats, ${ }^{\mathbf{1 0 2 4 b}}$ the primary capture antibodies or biotin molecules are applied typically in $\mathrm{mg} \mathrm{mL}^{-1}$ concentrations, several orders higher than fg $\mathrm{mL}^{-1}$ concentrations of target analytes. In addition to this main weakness of the direct assay format, the NTM substrate gives typically quite strong SERS blank background overlapping with characteristic spectral bands of reporters. As a result, only sandwich format should be used at the first immunoassay step, whereas the SERS readout with properly designed SERS tags can further enhance the overall assay sensitivity. Additionally, the use of nanoscaled surface shear flow ${ }^{50 c}$ or alternative current electrohydrodynamic (acEHD)-induced nanoscaled surface shear forces ${ }^{\mathbf{1 0}}$ enhances the capture kinetics and the binding specificity by eliminating nonspecifically bounded species.

The fabricated SERS tags with embedded different Raman molecules provide distinct spectral barcoding for detection and quantification for target analytes in a multiplexed format. For proof-of-concept illustration, a multiplexed detection of $\mathrm{H}-\mathrm{Ig} \mathrm{G}$, R-IgG, and Ch-IgG was performed in some analogy with previous approach based on multicolour staining with $\mathrm{Ag}-\mathrm{Au}$ nanocages tuned to blue, yellow, and red colours of solutions by variation of $\mathrm{Ag} / \mathrm{Au}$ ratio. ${ }^{45}$ Fig. 3a illustrates schematically the experiment principle. Three analytes were spotted on membrane squares designated by A\#, B\#, and C\# symbols; other squares were spotted with BSA as negative control. The analyte concentrations for spots (A2, B1, C4) and (A3, B5, C3) were $25 \mu \mathrm{g} \mathrm{mL} \mathrm{m}^{-1}$ and $100 \mu \mathrm{g} \mathrm{mL} \mathrm{m}^{-1}$, respectively. After staining with a mixture of GERT1, GERT2, and GERT3 conjugates (Fig. 3b) no nonspecific staining was observed and all analyte spots had, as expected, similar light-red colours because of negligible difference in plasmonic peaks of conjugates. However, due to unique distinct spectral peaks (Fig. 3d), the Raman mapping with PCA decomposition clearly demonstrates different false colours for corresponding analytes. Note that the intensity of colour spots,

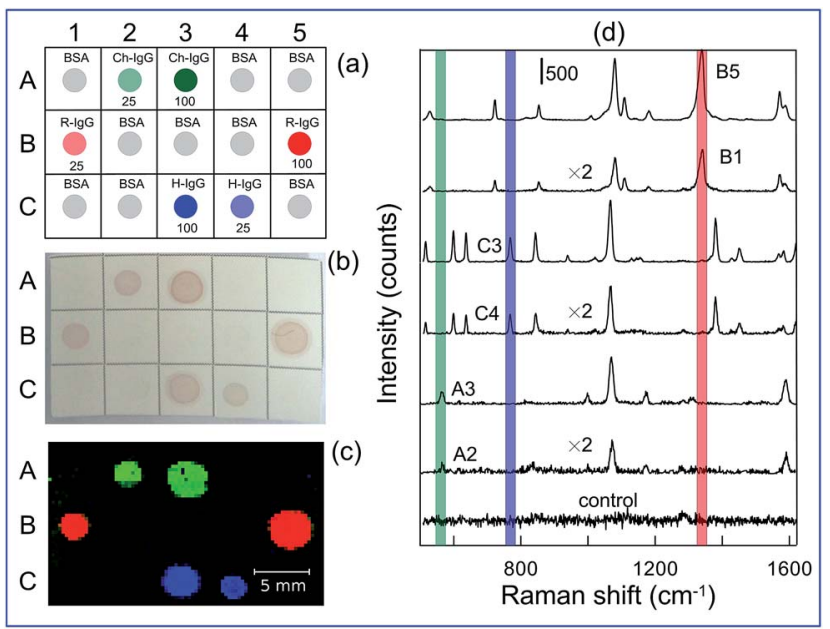

Fig. 3 (a) Schematic representation of multiplexed detection of R-IgG, $\mathrm{H}-\lg G$, and $\mathrm{Ch}-\lg \mathrm{G}$ analytes. The numbers below colour spots stand for concentration of analytes in $\mu \mathrm{g} \mathrm{mL}^{-1}$. BSA was used as negative control. (b) Visual coloration of analytes after staining in the mixture of GERT1, GERT2, and GERT3 conjugates. No difference in spot colour is seen for different analytes. (c) Multiplexed detection of three analytes by Raman mapping of membrane shown in the panel (b). The PCA analysis was used for false-colour imaging. The bar is $5 \mathrm{~mm}$. (d) SERS spectra recorded for spots with analytes A2, A3, B1, B5, C3, C4, and BSA control. The light red, blue, and green bars select characteristic barcode peaks at 1339,768 , and $583 \mathrm{~cm}^{-1}$ for spots $A(2,3), B(1,5)$, and $C(3,4)$, respectively. 
being derived from PCA decomposition, does not depend on the analyte concentration, as it should be for probability weight factors within the linear response regime. Therefore, one can easily discriminate between different analytes irrespectively of their concentrations. By contrast, the map of Raman intensity (Fig. S1, ESI $\dagger$ ) clearly demonstrates the difference in the colour brightness for different concentrations.

\section{Conclusions}

In this work, we have demonstrated a multiplex version of a direct SERS immunoassay based on a new type of gapenhanced Raman tags. Because of superior intrinsic properties of GERTs, they can be easily used in all modifications of SERS immunoassay that utilize sandwich format which sensitivity and specificity can be additionally enhanced by nanoscaled surface shear flow. Due to internal location of Raman reporters inside a plasmonic tag, they produce stable and intense SERS signals independently of local environment conditions and aggregation state. This property is crucial for SERS readout of whole Raman maps or selected points on substrates. Further, the outer size of GERTs can be reduced to 35-40 $\mathrm{nm}$ through formation of a thin Ag layer around thiolated Raman reporters attached to a small (10-15 $\mathrm{nm})$ gold core. In principle, such SERS tags can be used in SERS-based versions of immunochromatographic assays in evident analogy with $45 \mathrm{~nm}$ hollow gold nanospheres ${ }^{47}$ as well as in other solid-phase immunoassays utilizing core-shell particles and labels. ${ }^{51}$ Finally, the gap-enhanced Raman tags can be incorporated in the development of commercially available, hand-held, sensitive readers for evaluation of quantitative results and the integration into systems designed to optimize the performance of SERS-based immunoassays. ${ }^{47}$

\section{Conflicts of interest}

There are no conflicts to declare.

\section{Acknowledgements}

This work was supported by Russian Foundation for Basic Research (projects no. 16-02-00054, 16-52-45026, and 17-0200075). The work by V. A. Khanadeev was supported by a grant from the President of Russian Federation (project no. MK2617.2017.2). The work by T. E. Pylaev (functionalization of GERTs) was supported by a grant from the Russian Scientific Foundation (project no. 17-74-10090).

\section{Notes and references}

1 (a) M. D. Porter, R. J. Lipert, L. M. Siperko, G. Wang and R. Narayanan, Chem. Soc. Rev., 2008, 37, 1001; (b) J. Smolsky, S. Kaur, C. Hayashi, S. K. Batra and A. V. Krasnoslobodtsev, Biosensors, 2017, 7(1), 7.

2 (a) X. X. Han, L. Chen, W. Ji, Y. Xie, B. Zhao and Y. Ozaki, Small, 2011, 7, 316; (b) D. Lin, J. Pan, H. Huang, G. Chen,
S. Qiu, H. Shi, W. Chen, Y. Yu, S. Feng and R. Chen, Sci. Rep., 2014, 4, 4751.

3 K. V. Kong, W. K. Leong, Z. Lam, T. Gong, D. Goh, W. K. O. Lau and M. Olivo, Small, 2014, 10, 5030.

4 K. W. Kho, U. S. Dinish, A. Kumar and M. Olivo, ACS Nano, 2012, 6, 4892.

5 M. Kahraman, I. Sur and M. Culha, Anal. Chem., 2010, 82, 7596.

6 (a) X. Su, J. Zhang, L. Sun, T.-W. Koo, S. Chan, N. Sundararajan, M. Yamakawa and A. A. Berlin, Nano Lett., 2005, 5, 49; (b) J. D. Driskell, K. M. Kwarta, R. J. Lipert, M. D. Porter, J. D. Neill and J. F. Ridpath, Anal. Chem., 2005, 77, 6147; (c) W. E. Doering, M. E Piotti, M. J. Natan and R. G. Freeman, Adv. Mater., 2007, 19, 3100. 7 Y. Wang, B. Yan and L. Chen, Chem. Rev., 2013, 113, 1391.

8 W. Xie and S. Schlücker, Phys. Chem. Chem. Phys., 2013, 15, 5329.

9 (a) A. Wang, W. Ruan, W. Song, L. Chen, B. Zhao, Y. M. Jung and X. Wang, J. Raman Spectrosc., 2013, 44, 1649; (b) A. Yang, D. Wang, X. Wang, Yu. Han, X. Ke, H.-J. Wang, X. Zhou and L. Ren, $R S C A d v$., 2015, 5, 38354; (c) M. Li, J. W. Kang, S. Sukumar, R. R. Dasari and I. Barman, Chem. Sci., 2015, 6, 3906; (d) B. Tang, J. Wang, J. A. Hutchison, L. Ma, N. Zhang, H. Guo, Z. Hu, M. Li and Y. Zhao, ACS Nano, 2016, 10, 871; (e) L. Zhou, J. Zhou, Z. Feng, F. Wang, S. Xie and S. Bu, Analyst, 2016, 141, 2534; (f) X. Wang, L. Zhou, G. Wei, T. Jiang and J. Zhou, RSC Adv., 2016, 6, 708; $(g)$ X. Li, Y. Zhang, B. Xuea, X. Kong, X. Liu, L. Tu, Y. Chang and H. Zhang, Biosens. Bioelectron., 2017, 92, 517; $(h)$ L. Yang, M. X. Gao, L. Zhan, M. Gong, S. J. Zhen and C. Z. Huang, Nanoscale, 2017, 9, 2640.

10 K. K. Reza, J. Wang, R. Vaidyanathan, S. Dey, Y. Wang and M. Trau, Small, 2017, 13, 1602902.

11 J. Moon, S. Y. Yi, A. Hwang, G. Eom, J. Sim, J. Jeong, E.-K. Lim, B. H. Chung, B. Kim and J. J. T. Kang, RSC Adv., 2016, 6, 84415.

12 J. Wang, X. Wu, C. Wang, Z. Rong, H. Ding, H. Li, S. Li, N. Shao, P. Dong, R. Xiao and S.-Q. Wang, ACS Appl. Mater. Interfaces, 2016, 8, 19958.

13 (a) H. Chon, R. Wang, S. Lee, S.-Y. Bang, H.-S. Lee, S.-C. Bae, S. H. Hong, Y. H. Yoon, D. W. Lim, A. J. deMello and J. Choo, Anal. Bioanal. Chem., 2015, 407, 8353; (b) H. Chon, S. Lee, R. Wang, S.-Y. Bang, H.-S. Lee, S.-C. Bae, H. Lee, B. Kim and J. Choo, RSC Adv., 2014, 4, 32924.

14 A. C. Crawford, L. B. Laurentius, T. S. Mulvihill, J. H. Granger, J. S. Spencer, D. Chatterjee, K. E. Hanson and M. D. Porter, Analyst, 2017, 142, 186.

15 T. Mostowtt and B. McCord, Talanta, 2017, 164, 396.

16 (a) L. He, N.-J. Kim, H. Li, Z. Hu and M. Lin, J. Agric. Food Chem., 2008, 56, 9843; (b) B. B. Dzantiev, N. A. Byzova, A. E. Urusov and A. V. Zherdev, TrAC, Trends Anal. Chem., 2014, 55, 81.

17 Y. Wang, S. Chen, C. Wei, M. Xu, J. Yao, Y. Li, A. Deng and R. Gua, Chem. Commun., 2014, 50, 9112.

18 S. W. Bishnoi, Y. Lin, M. Tibudan, Y. Huang, M. Nakaema, V. Swarup and T. A. Keiderling, Anal. Chem., 2011, 83, 4053. 
19 (a) D. Brada and J. Roth, Anal. Biochem., 1984, 142, 79; (b) M. Moeremans, G. Daneles, A. van Dijck, G. Langanger and J. De Mey, J. Immunol. Methods, 1984, 74, 353; (c) B. Surek and E. Latzko, Biochem. Biophys. Res. Commun., 1984, 121, 284; (d) Y.-H. Hsu, Anal. Biochem., 1984, 142, 221.

20 L. A. Dykman and V. A. Bogatyrev, Biochemistry, 1997, 62, 350.

21 A. Goldman, S. Harper and D. W. Speicher, Curr. Protoc. Protein Sci., 2016, 86, 10.8.1.

22 L. Dykman and N. Khlebtsov, Chem. Soc. Rev., 2012, 41, 2256.

23 (a) W. Lu, A. K. Singh, S. A. Khan, D. Senapati, H. Yu and P. C. Ray, J. Am. Chem. Soc., 2010, 132, 18103; (b) M. E. Pekdemir, D. Erturkan, H. Kulah, I. H. Boyaci, C. Ozgen and U. Tamer, Analyst, 2012, 137, 4834; (c) G. Wang, R. J. Lipert, M. Jain, S. Kaur, S. Chakraboty, M. P. Torres, S. K. Batra, R. E. Brand and M. D. Porter, Anal. Chem., 2011, 83, 2554; (d) C. Song, L. Min, N. Zhou, Y. Yang, S. Su, W. Huang and L. Wang, ACS Appl. Mater. Interfaces, 2014, 6(24), 21842; (e) M. Guo, J. Dong, W. Xie, L. Tao, W. Lu, Y. Wang and W. Qian, J. Mater. Sci., 2015, 50, 3329.

24 (a) Y. Wang, M. Salehi, M. Schutz and S. Schlucker, Chem. Commun., 2014, 50, 2711; (b) K. Karn-orachai, K. Sakamoto, R. Laocharoensuk, S. Bamrungsap, T. Dharakulcd and K. Miki, RSC Adv., 2017, 7, 14099.

25 M. Li, S. K. Cushing, J. Zhang, S. Suri, R. Evans, W. P. Petros, L. F. Gibson, D. Ma, Y. Liu and N. Wu, ACS Nano, 2013, 7, 4967; T. Buchner, D. Drescher, H. Traub, P. Schrade, S. Bachmann, N. Jakubowski and J. Kneipp, Anal. Bioanal. Chem., 2014, 406, 7003.

26 D. K. Lim, K. S. Jeon, J. H. Hwang, H. Kim, S. Kwon, Y. D. Suh and J. M. Nam, Nat. Nanotechnol., 2011, 6, 452.

27 N. Gandra and S. Singamaneni, Adv. Mater., 2013, 25, 1022.

28 (a) N. Gandra, C. Portz and S. Singamaneni, Adv. Mater., 2014, 26, 424; (b) K.-K. Liu, S. Tadepalli, L. Tian and S. Singamaneni, Chem. Mater., 2015, 27, 5261; (c) A. M. Fales and T. Vo-Dinh, J. Mater. Chem. C, 2015, 3, 7319; (d) N. Gandra, H. C. Hendargo, S. J. Norton, A. M. Fales, G. M. Palmer and T. Vo-Dinh, Nanoscale, 2016, 8, 8486; (e) H. T. Ngo, N. Gandra, A. M. Fales, S. M. Taylor and T. Vo-Dinh, Biosens. Bioelectron., 2016, 81, 8; $(f)$ W. Xiong, D. Sikdar, L. W. Yap, P. Guo, M. Premaratne, X. Li and W. Cheng, Nano Res., 2016, 9, 415.

29 Y. Zhang, Y. Qiu, L. Lin, H. Gu, Z. Xiao and J. Ye, ACS Appl. Mater. Interfaces, 2017, 9, 3995.

30 B. N. Khlebtsov and N. G. Khlebtsov, J. Phys. Chem. C, 2016, 120, 15385.

31 (a) J.-W. Oh, D.-K. Lim, G.-H. Kim, Y. D. Suh and J.-M. Nam, J. Am. Chem. Soc., 2014, 136, 14052; (b) L. Lin, M. Zapata, M. Xiong, Z. Liu, S. Wang, H. Xu, A. G. Borisov, H. Gu, P. Nordlander, J. Aizpurua and J. Ye, Nano Lett., 2015, 15, 6419.

32 N. G. Khlebtsov and B. N. Khlebtsov, J. Quant. Spectrosc. Radiat. Transfer, 2017, 190, 89.
33 (a) W. Kang, P. T. C. So, R. R. Dasari and D.-K. Lim, Nano Lett., 2015, 15, 1766; (b) J. Li, Z. Zhu, B. Zhu, Y. Ma, B. Lin, R. Liu, Y. Song, H. Lin, S. Tu and C. Yang, Anal. Chem., 2016, 88, 782.

34 T. Yang and J. Jiang, Small, 2016, 36, 4980.

35 (a) V. Amendola and M. Meneghetti, Adv. Funct. Mater., 2012, 22, 353; (b) B. Mir-Simon, I. Reche-Perez, L. Guerrini, N. Pazos-Perez and R. A. Alvarez-Puebla, Chem. Mater., 2015, 27, 950.

36 (a) E. B. Santos, F. A. Sigoli and I. O. Mazali, Vib. Spectrosc., 2013, 68, 246; (b) B. N. Khlebtsov, V. A. Khanadeev, E. V. Panfilova, D. N. Bratashov and N. G. Khlebtsov, ACS Appl. Mater. Interfaces, 2015, 7, 6518.

37 I. V. Yegorenkova, K. V. Tregubova, L. Yu. Matora, G. L. Burygin and V. V. Ignatov, Curr. Microbiol., 2010, 61, 376.

38 L. Lin, H. Gu and J. Ye, Chem. Commun., 2015, 51, 17740.

39 Y. Wang, A. B. Serrano, K. Sentosun, S. Bals and L. M. LizMarzan, Small, 2015, 11, 4314.

40 L. Tian, M. Fei, S. Tadepalli, J. J. Morrissey, E. D. Kharasch and S. Singamaneni, Adv. Healthcare Mater., 2015, 4, 1502.

41 M. E. Abdelsalam, Cent. Eur. J. Chem., 2009, 7, 446.

42 E. C. Le Ru, E. Blackie, M. Meyer and P. G. Etchegoin, J. Phys. Chem. C, 2007, 111, 13794.

43 H. Liao and J. H. Hafner, Chem. Mater., 2005, 17, 4636.

44 V. A. Khanadeev, B. N. Khlebtsov, S. A. Staroverov,

I. V. Vidyasheva, A. A. Skaptsov, E. S. Ileneva, V. A. Bogatyrev, L. A. Dykman and N. G. Khlebtsov, J. Biophotonics, 2011, 4, 74.

45 E. Panfilova, A. Shirokov, B. Khlebtsov, L. Matora and

N. Khlebtsov, Nano Res., 2012, 5, 124.

46 B. Khlebtsov and N. Khlebtsov, Nanotechnology, 2008, 19, 435703.

47 (a) J. Y. Liao and H. Li, Microchim. Acta, 2010, 171, 289; (b) K. Zhang, J. Wu, Y. Li, Y. Wu, T. Huang and D. Tang, Microchim. Acta, 2014, 181, 1447; (c) J. Hwang, S. Lee and J. Choo, Nanoscale, 2016, 8, 11418.

48 A. C. Crawford, A. Skuratovsky and M. D. Porter, Anal. Chem., 2016, 88, 6515.

49 (a) R. Que, M. Shao, S. Zhuo, C. Wen, S. Wang and S.-T. Lee, Adv. Funct. Mater., 2011, 21, 3337; (b) X. Zhou, F. Zhou, H. Liu, L. Yang and J. Liu, Analyst, 2013, 138, 5832.

50 (a) Y. Chen, H. Cheng, K. Tram, S. Zhang, Y. Zhao, L. Han, Z. Chen and S. Huan, Analyst, 2013, 138, 2624; (b) T. Jiang, L. Zhang and J. Zhou, Analyst, 2014, 139, 5893; (c) Y. Wang, R. Vaidyanathan, M. J. A. Shiddiky and M. Trau, ACS Nano, 2015, 9, 6354.

51 (a) Q. Zhou, Y. Lin, M. Xu, Z. Gao, H.-H. Yang and D. Tang, Anal. Chem., 2016, 88, 8886; (b) Q. Zhou, Y. Lin, Y. Lin, Q. Wei, G. Chen and D. Tang, Biosens. Bioelectron., 2016, 78, 236; (c) Z. Qiu, J. Shu and D. Tang, Anal. Chem., 2017, 89, 5152; (d) K. Zhang, S. Lv, Z. Lin and D. Tang, Biosens. Bioelectron., 2017, 95, 34. 